\title{
Asymptotically ideal invariant equivalence
}

\section{UĞUR ULUSU}

ABSTRACT. In this paper, the concepts of asymptotically $\mathcal{I}_{\sigma}$-equivalence, $\sigma$-asymptotically equivalence, strongly $\sigma$-asymptotically equivalence and strongly $\sigma$-asymptotically $p$-equivalence for real number sequences are defined. Also, we give relationships among these new type equivalence concepts and the concept of $S_{\sigma}$-asymptotically equivalence which is studied in [Savaş, E. and Patterson, R. F., $\sigma$-asymptotically lacunary statistical equivalent sequences, Cent. Eur. J. Math., 4 (2006), No. 4, 648-655]

\section{INTRODUCTION AND BACKGROUND}

Let $\sigma$ be a mapping of the positive integers into themselves. A continuous linear functional $\phi$ on $\ell_{\infty}$, the space of real bounded sequences, is said to be an invariant mean or a $\sigma$-mean if it satisfies following conditions:

(1) $\phi(x) \geq 0$, when the sequence $x=\left(x_{n}\right)$ has $x_{n} \geq 0$ for all $n$,

(2) $\phi(e)=1$, where $e=(1,1,1, \ldots)$ and

(3) $\phi\left(x_{\sigma(n)}\right)=\phi\left(x_{n}\right)$ for all $x \in \ell_{\infty}$.

The mappings $\sigma$ are assumed to be one-to-one and such that $\sigma^{m}(n) \neq n$ for all positive integers $n$ and $m$, where $\sigma^{m}(n)$ denotes the $m$ th iterate of the mapping $\sigma$ at $n$. Thus, $\phi$ extends the limit functional on $c$, the space of convergent sequences, in the sense that $\phi(x)=\lim x$ for all $x \in c$.

In the case $\sigma$ is translation mappings $\sigma(n)=n+1$, the $\sigma$-mean is often called a Banach limit and the space $V_{\sigma}$, the set of bounded sequences all of whose invariant means are equal, is the set of almost convergent sequences $\hat{c}$.

It can be shown that

$$
V_{\sigma}=\left\{x=\left(x_{n}\right) \in \ell_{\infty}: \lim _{m \rightarrow \infty} \frac{1}{m} \sum_{k=1}^{m} x_{\sigma^{k}(n)}=L, \text { uniformly in } n\right\} .
$$

Several authors have studied invariant convergent sequences (see, [5-9,12-14,16, 18]). The concept of strongly $\sigma$-convergence was defined by Mursaleen in [6] as follows:

A bounded sequence $x=\left(x_{k}\right)$ is said to be strongly $\sigma$-convergent to $L$ if

$$
\lim _{m \rightarrow \infty} \frac{1}{m} \sum_{k=1}^{m}\left|x_{\sigma^{k}(n)}-L\right|=0,
$$

uniformly in $n$. It is denoted by $x_{k} \rightarrow L\left[V_{\sigma}\right]$.

By $\left[V_{\sigma}\right]$, we denote the set of all strongly $\sigma$-convergent sequences. In the case $\sigma(n)=n+1$, the space $\left[V_{\sigma}\right]$ is the set of strongly almost convergent sequences $[\hat{c}]$. 
The concept of strongly $\sigma$-convergence was generalized by Savaş [13] as below:

$$
\left[V_{\sigma}\right]_{p}=\left\{x=\left(x_{k}\right): \lim _{m \rightarrow \infty} \frac{1}{m} \sum_{k=1}^{m}\left|x_{\sigma^{k}(n)}-L\right|^{p}=0, \text { uniformly in } n\right\},
$$

where $0<p<\infty$.

If $p=1$, then $\left[V_{\sigma}\right]_{p}=\left[V_{\sigma}\right]$. It is known that $\left[V_{\sigma}\right]_{p} \subset \ell_{\infty}$.

The idea of statistical convergence was introduced by Fast [1] and studied by many authors.

A sequence $x=\left(x_{k}\right)$ is said to be statistically convergent to $L$ if for every $\varepsilon>0$

$$
\lim _{n \rightarrow \infty} \frac{1}{n}\left|\left\{k \leq n:\left|x_{k}-L\right| \geq \varepsilon\right\}\right|=0,
$$

where the vertical bars indicate the number of elements in the enclosed set.

The concept of $\sigma$-statistically convergent sequence was introduced by Savaş and Nuray in [16] as follows:

A sequence $x=\left(x_{k}\right)$ is $\sigma$-statistically convergent to $L$ if for every $\varepsilon>0$

$$
\lim _{m \rightarrow \infty} \frac{1}{m}\left|\left\{k \leq m:\left|x_{\sigma^{k}(n)}-L\right| \geq \varepsilon\right\}\right|=0,
$$

uniformly in $n$. It is denoted by $S_{\sigma}-\lim x=L$ or $x_{k} \rightarrow L\left(S_{\sigma}\right)$.

The idea of $\mathcal{I}$-convergence was introduced by Kostyrko et al. [3] as a generalization of statistical convergence which is based on the structure of the ideal $\mathcal{I}$ of subset of the set of natural numbers $\mathbb{N}$. Similar concepts can be seen in $[2,9]$.

A family of sets $\mathcal{I} \subseteq 2^{\mathbb{N}}$ is called an ideal if and only if $(i) \emptyset \in \mathcal{I}$, (ii) For each $A, B \in \mathcal{I}$ we have $A \cup B \in \mathcal{I}$, (iii) For each $A \in \mathcal{I}$ and each $B \subseteq A$ we have $B \in \mathcal{I}$.

An ideal is called non-trivial if $\mathbb{N} \notin \mathcal{I}$ and non-trivial ideal is called admissible if $\{n\} \in \mathcal{I}$ for each $n \in \mathbb{N}$.

All ideals in this paper are assumed to be admissible.

A family of sets $\mathcal{F} \subseteq 2^{\mathbb{N}}$ is called a filter if and only if $(i) \emptyset \notin \mathcal{F},(i i)$ For each $A, B \in \mathcal{F}$ we have $A \cap B \in \mathcal{F}$, (iii) For each $A \in \mathcal{F}$ and each $B \supseteq A$ we have $B \in \mathcal{F}$.

For any ideal there is a filter $\mathcal{F}(\mathcal{I})$ corresponding with $\mathcal{I}$, given by

$$
\mathcal{F}(\mathcal{I})=\{M \subset \mathbb{N}:(\exists A \in \mathcal{I})(M=\mathbb{N} \backslash A)\} .
$$

A sequence $x=\left(x_{k}\right)$ is said to be $\mathcal{I}$-convergent to $L$ if for every $\varepsilon>0$, the set

$$
A(\varepsilon)=\left\{k \in \mathbb{N}:\left|x_{k}-L\right| \geq \varepsilon\right\},
$$

belongs to $\mathcal{I}$. If $x=\left(x_{k}\right)$ is $\mathcal{I}$-convergent to $L$, then we write $\mathcal{I}-\lim x=L$.

Recently, the concepts of $\sigma$-uniform density of subset $A$ of the set $\mathbb{N}$ of positive integers and corresponding $\mathcal{I}_{\sigma}$-convergence for real number sequences was introduced by Nuray et al. [9]. 
Let $A \subseteq \mathbb{N}$ and

$$
s_{m}=\min _{n}\left|A \cap\left\{\sigma(n), \sigma^{2}(n), \ldots, \sigma^{m}(n)\right\}\right| \text { and } S_{m}=\max _{n}\left|A \cap\left\{\sigma(n), \sigma^{2}(n), \ldots, \sigma^{m}(n)\right\}\right| .
$$

If the following limits exists

$$
\underline{V}(A)=\lim _{m \rightarrow \infty} \frac{s_{m}}{m}, \quad \bar{V}(A)=\lim _{m \rightarrow \infty} \frac{S_{m}}{m},
$$

then they are called a lower $\sigma$-uniform density and an upper $\sigma$-uniform density of the set $A$, respectively.

If $\underline{V}(A)=\bar{V}(A)$, then $V(A)=\underline{V}(A)=\bar{V}(A)$ is called the $\sigma$-uniform density of $A$.

Denote by $\mathcal{I}_{\sigma}$ the class of all $A \subseteq \mathbb{N}$ with $V(A)=0$.

Throughout the paper we take $\mathcal{I}_{\sigma}$ as an admissible ideal in $\mathbb{N}$.

A sequence $x=\left(x_{k}\right)$ is said to be $\mathcal{I}_{\sigma}$-convergent to $L$ if for every $\varepsilon>0$, the set

$$
A_{\varepsilon}=\left\{k:\left|x_{k}-L\right| \geq \varepsilon\right\},
$$

belongs to $\mathcal{I}_{\sigma}$; i.e., $V\left(A_{\varepsilon}\right)=0$. It is denoted by $\mathcal{I}_{\sigma}-\lim x_{k}=L$.

Marouf [4] presented definitions for asymptotically equivalent sequences and asymptotic regular matrices. Then, the concept of asymptotically equivalence has been developed by many other researchers (see, $[10,11,15,17])$.

Two nonnegative sequences $x=\left(x_{k}\right)$ and $y=\left(y_{k}\right)$ are said to be asymptotically equivalent if

$$
\lim _{k} \frac{x_{k}}{y_{k}}=1 \text {. }
$$

It is denoted by $x \sim y$.

Two nonnegative sequences $x=\left(x_{k}\right)$ and $y=\left(y_{k}\right)$ are $S_{\sigma}$-asymptotically equivalent of multiple $L$ provided that for every $\varepsilon>0$

$$
\lim _{n} \frac{1}{n}\left|\left\{k \leq n:\left|\frac{x_{\sigma^{k}(m)}}{y_{\sigma^{k}(m)}}-L\right| \geq \varepsilon\right\}\right|=0,
$$

uniformly in $m=1,2, \ldots$, (denoted by $x \stackrel{S_{\sigma}}{\sim} y$ ) and simply $S_{\sigma}$-asymptotically statistical equivalent, if $L=1$.

\section{ASYMPtoticAlly $\mathcal{I}_{\sigma}$-EQUiVALENCE}

In this section, the concepts of asymptotically $\mathcal{I}_{\sigma}$-equivalence, $\sigma$-asymptotically equivalence, strongly $\sigma$-asymptotically equivalence and strongly $\sigma$-asymptotically $p$-equivalence for real number sequences are defined. Also, we examine relationships among these new type equivalence concepts and the concept of $S_{\sigma}$-asymptotically equivalence which is studied in this area before.

Definition 2.1. Two nonnegative sequences $x=\left(x_{k}\right)$ and $y=\left(y_{k}\right)$ are said to be asymptotically $\mathcal{I}_{\sigma}$-equivalent of multiple $L$ if for every $\varepsilon>0$

$$
A_{\varepsilon}:=\left\{k \in \mathbb{N}:\left|\frac{x_{k}}{y_{k}}-L\right| \geq \varepsilon\right\} \in \mathcal{I}_{\sigma} ;
$$

i.e., $V\left(A_{\varepsilon}\right)=0$. In this case, we write $x \stackrel{\mathcal{I}_{\sigma}^{L}}{\sim} y$ and simply asymptotically $\mathcal{I}_{\sigma}$-equivalent, if $L=1$. $\mathfrak{I}_{\sigma}^{L}$.

The set of all asymptotically $\mathcal{I}_{\sigma}$-equivalent of multiple $L$ sequences will be denoted by 
Definition 2.2. Two nonnegative sequence $x=\left(x_{k}\right)$ and $y=\left(y_{k}\right)$ are $\sigma$-asymptotically equivalent of multiple $L$ if

$$
\lim _{n \rightarrow \infty} \frac{1}{n} \sum_{k=1}^{n} \frac{x_{\sigma^{k}(m)}}{y_{\sigma^{k}(m)}}=L
$$

uniformly in $m$. In this case, we write $x \stackrel{V_{\sigma}^{L}}{\sim} y$ and simply $\sigma$-asymptotically equivalent, if $L=1$.

Theorem 2.1. Suppose that $x=\left(x_{k}\right)$ and $y=\left(y_{k}\right)$ are bounded sequences. If $x$ and $y$ are asymptotically $\mathcal{I}_{\sigma}$-equivalent of multiple $L$, then these sequences are $\sigma$-asymptotically equivalent of multiple $L$.

Proof. Let $m, n \in \mathbb{N}$ be an arbitrary and $\varepsilon>0$. Now, we calculate

$$
t(m, n):=\left|\frac{1}{n} \sum_{k=1}^{n} \frac{x_{\sigma^{k}(m)}}{y_{\sigma^{k}(m)}}-L\right|
$$

We have

$$
t(m, n) \leq t^{(1)}(m, n)+t^{(2)}(m, n)
$$

where

$$
\begin{aligned}
& t^{(1)}(m, n):=\frac{1}{n} \quad \sum_{k=1}^{n} \quad\left|\frac{x_{\sigma^{k}(m)}}{y_{\sigma^{k}(m)}}-L\right| \text { and } t^{(2)}(m, n):=\frac{1}{n} \sum_{k=1}^{n}\left|\frac{x_{\sigma^{k}(m)}}{y_{\sigma^{k}(m)}}-L\right| \text {. } \\
& \left|\frac{x_{\sigma^{k}(m)}}{y_{\sigma^{k}(m)}}-L\right| \geq \varepsilon \quad \mid \frac{x_{\sigma^{k}(m)}}{y_{\sigma^{k}(m)}(m \mid<\varepsilon}
\end{aligned}
$$

We get $t^{(2)}(m, n)<\varepsilon$, for every $m=1,2, \ldots$. The boundedness of $x=\left(x_{k}\right)$ and $y=\left(y_{k}\right)$ implies that there exists a $M>0$ such that

$$
\left|\frac{x_{\sigma^{k}(m)}}{y_{\sigma^{k}(m)}}-L\right| \leq M
$$

for $k=1,2, \ldots ; m=1,2, \ldots$. Then, this implies that

$$
\begin{aligned}
t^{(1)}(m, n) & \leq \frac{M}{n}\left|\left\{1 \leq k \leq n:\left|\frac{x_{\sigma^{k}(m)}}{y_{\sigma^{k}(m)}}-L\right| \geq \varepsilon\right\}\right| \\
& \leq M \frac{\max _{m}\left|\left\{1 \leq k \leq n:\left|\frac{x_{\sigma^{k}(m)}}{y_{\sigma^{k}(m)}}-L\right| \geq \varepsilon\right\}\right|}{n}=M \frac{S_{n}}{n},
\end{aligned}
$$

hence $x$ and $y$ are $\sigma$-asymptotically equivalent to multiple $L$.

The converse of Theorem 2.1 does not hold. For example, $x=\left(x_{k}\right)$ and $y=\left(y_{k}\right)$ are the sequences defined by following;

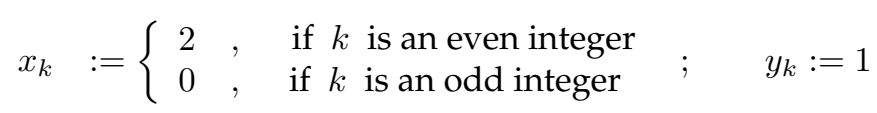

When $\sigma(m)=m+1$, this sequence is $\sigma$-asymptotically equivalent but it is not asymptotically $\mathcal{I}_{\sigma}$-equivalent.

Definition 2.3. Two nonnegative sequence $x=\left(x_{k}\right)$ and $y=\left(y_{k}\right)$ are strongly $\sigma$-asymptotically equivalent of multiple $L$ if

$$
\lim _{n \rightarrow \infty} \frac{1}{n} \sum_{k=1}^{n}\left|\frac{x_{\sigma^{k}(m)}}{y_{\sigma^{k}(m)}}-L\right|=0
$$


uniformly in $m$. In this case, we write $x \stackrel{\left[V_{\sigma}^{L}\right]}{\sim} y$ and simply strongly $\sigma$-asymptotically equivalent, if $L=1$.

Definition 2.4. Let $0<p<\infty$. Two nonnegative sequence $x=\left(x_{k}\right)$ and $y=\left(y_{k}\right)$ are strongly $\sigma$-asymptotically $p$-equivalent of multiple $L$ if

$$
\lim _{n \rightarrow \infty} \frac{1}{n} \sum_{k=1}^{n}\left|\frac{x_{\sigma^{k}(m)}}{y_{\sigma^{k}(m)}}-L\right|^{p}=0
$$

uniformly in $m$. In this case, we write $x \stackrel{\left[V_{\sigma}^{L}\right]_{p}}{\sim} y$ and simply strongly $\sigma$-asymptotically $p$-equivalent, if $L=1$.

The set of all strongly $\sigma$-asymptotically $p$-equivalent of multiple $L$ sequences will be denoted by $\left[\mathfrak{V}_{\sigma}^{L}\right]_{p}$.

Theorem 2.2. Let $0<p<\infty$. Then, $x \stackrel{\left[V_{\sigma}^{L}\right]_{p}}{\sim} y \Rightarrow x \stackrel{\mathcal{I}_{\sigma}^{L}}{\sim} y$.

Proof. Let $x \stackrel{\left[V_{\sigma}^{L}\right]_{p}}{\sim} y$ and given $\varepsilon>0$. Then, for every $m \in \mathbb{N}$ we have

$$
\begin{aligned}
\sum_{k=1}^{n}\left|\frac{x_{\sigma^{k}(m)}}{y_{\sigma^{k}(m)}}-L\right|^{p} & \geq \sum_{k=1}^{n}\left|\frac{x_{\sigma^{k}(m)}}{y_{\sigma^{k}(m)}}-L\right|^{p} \geq \varepsilon^{p} \cdot\left|\left\{1 \leq k \leq n:\left|\frac{x_{\sigma^{k}(m)}}{y_{\sigma^{k}(m)}}-L\right| \geq \varepsilon\right\}\right| \\
& \geq \varepsilon^{p} \cdot \max _{m} \mid\left\{1 \leq k \leq n:\left|\frac{x_{\sigma^{k}(m)}(m)}{y_{\sigma^{k}(m)}}-L\right| \geq \varepsilon\right. \\
y_{\sigma^{k}(m)} & |\{L \mid \geq \varepsilon\}|
\end{aligned}
$$

and

$$
\frac{1}{n} \sum_{k=1}^{n}\left|\frac{x_{\sigma^{k}(m)}}{y_{\sigma^{k}(m)}}-L\right|^{p} \geq \varepsilon^{p} \cdot \frac{\max _{m}\left|\left\{1 \leq k \leq n:\left|\frac{x_{\sigma^{k}(m)}}{y_{\sigma^{k}(m)}}-L\right| \geq \varepsilon\right\}\right|}{n}=\varepsilon^{p} \cdot \frac{S_{n}}{n}
$$

for every $m=1,2, \ldots$. This implies $\lim _{n \rightarrow \infty} \frac{S_{n}}{n}=0$ and so $x \stackrel{\mathcal{I}_{\sigma}^{L}}{\sim} y$.

Theorem 2.3. Let $0<p<\infty$ and $x, y \in \ell_{\infty}$. Then, $x \stackrel{\mathcal{I}_{\sigma}^{L}}{\sim} y \Rightarrow x \stackrel{\left[V_{\sigma}^{L}\right]_{p}}{\sim} y$.

Proof. Suppose that $x, y \in \ell_{\infty}$ and $x \stackrel{\mathcal{I}_{\sigma}^{L}}{\sim} y$. Let $\varepsilon>0$. By assumption, we have $V\left(A_{\varepsilon}\right)=0$. The boundedness of $x$ and $y$ implies that there exists a $M>0$ such that

$$
\left|\frac{x_{\sigma^{k}(m)}}{y_{\sigma^{k}(m)}}-L\right| \leq M
$$

for $k=1,2, \ldots ; m=1,2, \ldots$. Observe that, for every $m \in \mathbb{N}$ we have

$$
\begin{aligned}
\frac{1}{n} \sum_{k=1}^{n}\left|\frac{x_{\sigma^{k}(m)}}{y_{\sigma^{k}(m)}}-L\right|^{p} & =\frac{1}{n} \sum_{k=1}^{n}\left|\frac{x_{\sigma^{k}(m)}}{y_{\sigma^{k}(m)}}-L\right|^{p}+\frac{1}{n} \sum_{k=1}^{n}\left|\frac{x_{\sigma^{k}(m)}}{y_{\sigma^{k}(m)}}-L\right|^{p} \\
& \leq M \frac{\left.\max _{m}\right|_{\sigma^{k}(m)} ^{y_{\sigma^{k}(m)}}-L \mid \geq \varepsilon}{y_{\sigma^{k}(m)}(m)}-L \mid<\varepsilon \\
& \leq M \frac{S_{n}}{n}+\varepsilon^{p} .
\end{aligned}
$$


Hence, we obtain

uniformly in $m$.

$$
\lim _{n \rightarrow \infty} \frac{1}{n} \sum_{k=1}^{n}\left|\frac{x_{\sigma^{k}(m)}}{y_{\sigma^{k}(m)}}-L\right|^{p}=0
$$

Theorem 2.4. Let $0<p<\infty$. Then, $\mathfrak{I}_{\sigma}^{L} \cap \ell_{\infty}=\left[\mathfrak{V}_{\sigma}^{L}\right]_{p} \cap \ell_{\infty}$.

Proof. This is an immediate consequence of Theorem 2.2 and Theorem 2.3.

Now we shall state a theorem that gives a relationship between asymptotically $\mathcal{I}_{\sigma}$-equivalence and $S_{\sigma}$-asymptotically equivalence.

Theorem 2.5. The sequences $x=\left(x_{k}\right)$ and $y=\left(y_{k}\right)$ are asymptotically $\mathcal{I}_{\sigma}$-equivalent to multiple $L$ if and only if they are $S_{\sigma}$-asymptotically equivalent of multiple $L$.

\section{REFERENCES}

[1] Fast, H., Sur la convergence statistique, Colloq. Math., 2 (1951), 241-244

[2] Kostyrko, P., Macaj, M., Šalát, T. and Sleziak, M., I-Convergence and Extermal I-limits points, Math. Slovaca, 55 (2005), 443-464

[3] Kostyrko, P., Šalát, T. and Wilczyński, W., I-Convergence, Real Anal. Exchange, 26 (2000), No. 2, 669-686

[4] Marouf, M., Asymptotic equivalence and summability, Int. J. Math. Math. Sci., 16 (1993), No. 4, 755-762

[5] Mursaleen, M., On finite matrices and invariant means, Indian J. Pure Appl. Math., 10 (1979), 457-460

[6] Mursaleen, M., Matrix transformation between some new sequence spaces, Houston J. Math., 9 (1983), 505-509

[7] Mursaleen, M. and Edely, O. H. H., On the invariant mean and statistical convergence, Appl. Math. Lett., 22 (2009), No. 11, 1700-1704

[8] Nuray, F. and Savaş, E., Invariant statistical convergence and A-invariant statistical convergence, Indian J. Pure Appl. Math., 25 (1994), No. 3, 267-274

[9] Nuray, F., Gök, H. and Ulusu, U., $\mathcal{I}_{\sigma}$-convergence, Math. Commun., 16 (2011), 531-538

[10] Patterson, R. F., On asymptotically statistically equivalent sequences, Demostratio Math., 36 (2003), No. 1, 149-153

[11] Patterson, R. F. and Savaş, E., On asymptotically lacunary statistically equivalent sequences, Thai J. Math., 4 (2006), No. 2, 267-272

[12] Raimi, R. A., Invariant means and invariant matrix methods of summability, Duke Math. J., 30 (1963), No. 1, 81-94

[13] Savaş, E., Some sequence spaces involving invariant means, Indian J. Math., 31 (1989), 1-8

[14] Savaş, E., Strongly $\sigma$-convergent sequences, Bull. Calcutta Math., 81 (1989), 295-300

[15] Savaş, E., On I-asymptotically lacunary statistical equivalent sequences, Adv. Difference Equ., 111 (2013), 7 pages. doi:10.1186/1687-1847-2013-111

[16] Savaş, E. and Nuray, F., On $\sigma$-statistically convergence and lacunary $\sigma$-statistically convergence, Math. Slovaca, 43 (1993), No. 3, 309-315

[17] Savas,, E. and Patterson, R. F., $\sigma$-asymptotically lacunary statistical equivalent sequences, Cent. Eur. J. Math., 4 (2006), No. 4, 648-655

[18] Schaefer, P., Infinite matrices and invariant means, Proc. Amer. Math. Soc., 36 (1972), 104-110

DEPARTMENT OF MATHEMATICS

AFYON KOCATEPE UNIVERSITY

03200 AFYONKARAHISAR, TURKEY

E-mail address: ulusulaku. edu.tr 